\title{
AFFECTIVE INSTABILITY IN PATIENTS WITH CHRONIC PAIN: A DIARY APPROACH
}

Silke Rost ${ }^{1,2, *}$, Dimitri M.L. Van Ryckeghem ${ }^{2}$, Peter Koval ${ }^{3,4}$, Stefan Sütterlin ${ }^{5,6,7}$, Claus Vögele ${ }^{1,6}$, Geert Crombez ${ }^{2}$

${ }^{1}$ Institute for Health and Behaviour, INSIDE, University of Luxembourg, Luxembourg

${ }^{2}$ Department of Experimental-Clinical and Health Psychology, Ghent University, Belgium

${ }^{3}$ School of Psychology, Australian Catholic University, Australia

${ }^{4}$ Research Group of Quantitative Psychology and Individual Differences, University of Leuven, Belgium

${ }^{5}$ Section of Psychology, Lillehammer University College, Norway

${ }^{6}$ Research Group Health Psychology, University of Leuven, Belgium

${ }^{7}$ Department of Psychosomatic Medicine, Division of Surgery and Clinical Neuroscience,

Oslo University Hospital - Rikshospitalet, Norway

Keywords: affective instability, positive affect, negative affect, chronic pain, daily outcomes

Running title: Affective instability and chronic pain

20 pages, 3 tables

* Corresponding author. Address: University of Luxembourg, 11, Porte des Sciences, 4366 Esch-surAlzette, Luxembourg. Tel: + 352466644 9249; fax: +352 466644 9535. E-mail address: silke.rost@uni.lu (S. Rost). 


\begin{abstract}
Affective instability, conceptualized as fluctuations in mood over time, has been related to ill-health and psychopathology. In this study we examined the role of affective instability upon daily pain outcomes in 70 chronic pain patients ( $M_{\text {age }}=49.7$ years; 46 females) using an end-of-day diary. During a baseline phase, patients completed self-reported questionnaires of pain severity, pain duration, disability, depression and anxiety. During a subsequent diary phase, patients filled out an electronic end-of-day diary over 14 consecutive days assessing daily levels of pain severity, disability, cognitive complaints, negative affect (NA) and positive affect (PA). Affective instability was operationalized as the mean square of successive differences (MSSD) in daily mood (separately for NA and PA), which takes into account the size of affective changes over consecutive days. Results indicated that NA instability was positively associated with daily disability, beyond the effects of daily pain severity. Furthermore, NA instability moderated the relationship between daily pain severity and daily disability and the relationship between daily pain severity and daily cognitive complaints. PA instability, however showed to be unrelated to all outcomes. Current findings extend previous results and reveal the putative role of affective instability upon pain-related outcomes and may yield important clinical implications. Indeed, they suggest that targeting NA instability by improving emotion regulation skills may be a strategy to diminish disability and cognitive complaints in patients with chronic pain.
\end{abstract}




\section{Introduction}

Emotions change following the ebb-and-flow of daily life. However, experiencing unusually large and/or frequent changes in emotion, labeled affective instability, can be considered dysfunctional $[55,56]$. Affective instability may reflect problems in regulating affect $[8,47]$, which is a key feature of mental disorders [20]. In line with this view, affective instability has been found to be associated with lower wellbeing and various forms of psychopathology $[15,25,55,56]$. To our knowledge, there are no studies that have investigated the role of affective instability in patients with chronic pain.

There is abundant research demonstrating that patients with chronic pain report high levels of negative affect (NA) and low levels of positive affect (PA) on self-report questionnaires $[1,41,62]$. Questionnaire research, however, does not capture well the temporal dynamics of affect. Indeed, chronic pain patients experience fluctuations not only in pain [49], but also in NA and PA [52,63,64].

Investigating moment-to-moment variations in affect may further our understanding of chronic pain and associated problems. As an example, Sturgeon and colleagues (2014) showed that the relationships between daily PA and pain as well as daily NA and pain were mediated by positive interpersonal events and pain catastrophizing [53]. Similarly, Connelly and colleagues (2007) found that changes in PA and NA from the prior day to the current day predicts significantly greater decreases in pain that day [12]. In the current study, we examined the potential role of affective instability, an index that captures both the frequency and size of affective fluctuations [26], upon pain and its outcomes. This is in line with previous research which suggests that affect regulation may be a key factor for the adjustment to pain [22].

Affective instability may be considered an indicator of dysregulated affect [8,47]. As pain is conceptualized as a sensory and emotional experience, affective instability is likely to be closely related to the dysfunctional regulation of pain. With increasing affective instability, we may expect pain to interfere more with daily activities and cognitive processes [2,17,57]. Based on the results of a recent meta-analysis on the role of affective instability in psychological well-being [25], we expected NA instability to have a stronger influence on daily pain outcomes.

To investigate the role of affective instability, the current study assessed day-to-day fluctuations in levels of pain and mood using end-of-day diaries over 14 consecutive days. This method allowed us to investigate the impact of the dynamics of PA and NA on daily pain outcomes, i.e. disability and cognitive complaints. In particular, we were interested (1) to what extent NA and PA instability were related to standard measures of emotional functioning, such as anxiety and depression, (2) whether NA and PA instability were related to daily pain outcomes (disability and cognitive complaints), and (3) whether NA and PA instability moderated the relationship between daily pain severity on the one hand and daily disability and cognitive complaints on the other hand. 


\section{Method}

\subsection{Participants}

This study reports secondary analyses of data from the Ghent Pain Disability - I (GPD-I) Study. Previous research has been reported using a part of these data, but had other research objectives [see 59]. Detailed information on recruitment, primary research objectives and variables assessed can be found on the website (http://hdl.handle.net/1854/LU-3050986). We briefly report the most relevant information. 518 members of the Flemish Pain League responded to an invitation letter of which 315 agreed to be contacted by phone. 276 persons were actually contacted by phone. Patients were recruited in February and March 2011. Inclusion criteria were: 1) age between 18 and 65 years; 2) sufficient knowledge of the Dutch language; and 3) pain that lasted for at least six months. Individuals were excluded if headache pain was the most important pain [cfr. 36] ( $n=1)$, when they were unable to use both index fingers $(n=1)$ or when their eyesight was not normal or corrected-to-normal (e.g. by glasses) $(n=2)$. The latter two exclusion criteria were used as participants had to be able to perform a computer task. However, this task was not part of this study. 81 patients fulfilled the inclusion criteria and agreed to participate. The need to travel to the university campus to participate in this study was mentioned as the most frequent reason for nonparticipation. Seven more patients did not participate because of health problems. The final sample of patients consisted of 74 individuals with chronic pain.

Patients filled out some standard questionnaires (STAI-T, HADS-D, PDI, MPI) via an online assessment system at home. When arriving at the research lab, patients filled out the STAI-S. All these measures are labeled as "baseline" measures. Next, they participated in an experiment (see also 59) and received instructions for the diary study. The diary study started one day after the visit to the research lab. Patients filled out an online end-of-day diary for 14 consecutive days. Measures from the diary are labeled "daily" measures. The study design was approved by the Ethics Committee of the Faculty of Psychology and Educational Sciences of Ghent University. Patients provided written informed consent and received a monetary compensation for their participation.

\subsection{Questionnaires}

State and trait anxiety were measured with the State-Trait Anxiety Inventory (STAI) [51,58]. This questionnaire consists of 40 items asking people to report how they feel in general (e.g. I feel nervous) and at present (e.g. I feel at ease) using a scale from 1 (almost never/not at all) to 4 (almost always/very much so). Scores for the trait and state version may vary between 20 and 80 . This questionnaire shows good reliability and validity $[5,50]$. In the present study Cronbach's alpha was .93 for the trait version and .93 for the state version.

Depressive mood was assessed by means of the depression subscale of Hospital Anxiety and Depression Scale (HADS-D) [65]. The HADS-D is designed to screen for depression during the last week 
and has been developed for patients with "medical conditions". It consists of seven items rated on a 4point Likert scale. Scores may range from 0 to 21 . In the present study, Cronbach's alpha of the HADS-D was .81 .

Disability because of pain was measured with the Pain Disability Index (PDI) [40]. Patients indicate the degree of disability experienced in seven life domains (e.g. family and occupation) using a scale from 0 (no disability) to 10 (total disability). They are asked to respond to each category by indicating the overall impact of pain in their life, not just when pain is at its worst. Scores may vary between 0 and 70. In the present study, Cronbach's alpha of the PDI was .81.

Pain severity was assessed with the pain severity subscale of the Multidimensional Pain Inventory (MPI) [27,31]. Part I of the MPI consists of 5 subscales assessing the impact of pain (i.e. pain severity, pain interference, social support, perceived life control and affective distress) on a 7-point Likert scale. Pain severity was assessed by means of two items (i.e., "Rate the level of your pain at the present moment" and "On average, how severe has your pain been during the last week?"). The third item ("How much suffering do you experience because of your pain?") was not taken into account as its content relates to suffering rather than pain severity [see 39]. The MPI showed a good reliability and validity [45]. In the present study, Cronbach's alpha of the MPI severity subscale was .82 .

\subsection{End-of-day diary assessment}

Patients were asked to fill out an online diary at the end of each day for 2 consecutive weeks. They were reminded to do so each evening via a text message. The diary took approximately 5 minutes to complete. In this study, only the items which are of relevance for the current research aim are described. Items were developed iteratively by a group of pain researchers and piloted for feasibility in patients with chronic pain.

Positive Affect and Negative Affect: Items assessing daily PA and NA were rated on a scale from 0 (not at all agree) to 6 (totally agree). We used six adjectives for PA and NA respectively: glad [blij], enthusiastic [enthousiast], happy [gelukkig], relaxed [ontspannen], strong [sterk] and proud [trots] for PA and afraid [bang], irritated [geirriteerd], angry [kwaad], powerless [machteloos], sad [triest] and nervous [zenuwachtig] for NA. Items were derived from a validation study investigating the representation of emotion terms in a general population [60]. PA and NA scales were calculated by averaging PA and NA items respectively. We calculated within-person reliability of the PA and NA scales using the Generalizability theory approach described by Bolger and Laurenceau (2013) [7]. Estimates of withinperson reliability were .84 for PA and .77 for NA, indicating that both scales assessed within-person changes reliably. Pain severity was assessed using two items: "On average, how severe has your pain been today?" and "Which number would you ascribe to the pain you experienced the most today?" both rated 
on a scale from 0 (no pain) to 10 (worst imaginable pain). As the two pain items were highly correlated (within-person correlation was .78, $P<.001$ ), we calculated an average score of both items. Pain disability was assessed by the item “To what extent did pain interfere with your planned activities?" rated on a scale from 0 (not at all) to 10 (very much). This item corresponds to the PDI questioning, but asks more generally about the degree to which pain prevents patients from their planned activities. Cognitive complaints were assessed by three items: distractibility ("to what extent were you distracted today?"), forgetfulness ("to what extent were you forgetful today?") and clear thinking ("to what extent were you able to think clearly today?") all rated on a scale from 0 (not at all) to 10 (very much). The clear thinking item was reverse-coded (subtracting scores from 10) and scores on these three items were averaged to form a score for daily cognitive complaints with a range from 0 to 10 . The within-person reliability of the cognitive complaints scale was .80 . These items were selected based on previous research in which authors reported frequent cognitive problems of chronic pain patients $[34,44]$.

\subsection{Affective instability}

Affective instability, conceptualized as frequent mood shifts over time [26], takes into account the size of affective changes as well as their temporal order. It is related to the magnitude of the difference from one time-point to another. The mean square of successive differences (MSSD) has been proposed as an index of affective instability [26]. It reflects the extent to which consecutively assessed mood states differ from each other and, therefore, provides a measure of both variability (i.e., average magnitude of affective changes) and temporal dependency (i.e., average frequency of affective changes). In our study, this measure reflects both the frequency and size of fluctuations in daily mood over 14 days. The squared difference between successive observations at occasions $i+1$ and $i$ for a time series of $n$ measurement occasions is given by

$$
M S S D=\frac{1}{N-1} \sum_{i=1}^{N-1}\left(x_{i+1}-x_{i}\right)^{2}
$$

This index was calculated separately for PA and NA [see 25,28,55].

\subsection{Procedure and data handling}

Diary reports were included in the analyses only if they filled out the diary for at least 10 out of the 14 days. The data of four patients were excluded for this reason, providing a final sample of 70 patients. 


\section{6. $\quad$ Statistical model}

Descriptive statistics and correlation analyses were performed using SPSS statistical software, version 19.0 for Windows (SPSS Inc., Chicago/IL). The nested structure of the data (multiple observations nested within individuals) requires a multilevel regression approach. The HLM software package (Version 7.01; Scientific Software International, Skokie/IL) was used [42]. Level 1 variables consisted of the daily diary measures of affect (PA and NA), daily pain intensity, pain disability, and cognitive complaints. All Level 1 variables were continuous and entered group mean centered [19]. Level 2 variables consisted of baseline questionnaire measures of gender, age, pain duration, and baseline disability. Gender was dummy coded $(0=$ female; $1=$ male $)$ and entered uncentered, while all continuous Level 2 variables were standardized to facilitate interpretation [37]. Full maximum likelihood estimation was used for all models. We followed a model building procedure in our analyses [42]. To maximize stability and reliability of the findings, we excluded control variables from further steps in model building if their effects proved to be non-significant [29]. As suggested by some authors, we controlled for the mean level of daily affect in order to get the effect specific to within-individual variability $[16,46]$. Mean levels of daily PA and NA were therefore included in the final analyses at Level 2 . The moderating role of affective instability was investigated in the last step of model building. Models included random intercepts and random slopes.

\section{Results}

\subsection{Participant characteristics}

The average age of the patients was 49.7 years ( $S D=9.76$; range 22-64 years), and 46 were female $(65.7 \%)$. Most were married (63\%) or living together (10\%). A total of $42.6 \%$ reported a higher education level (college or university degree), $55.9 \%$ of the patients had a secondary school degree and the remaining $1.5 \%$ had a primary school degree. The sample consisted of a mixed group of chronic pain patients. All of them were seeking for help, taking medication, receiving some kind of pain treatment (e.g. occupational therapy, chiropractic, manual therapy) and/or seeing a specialist (e.g. orthopedist, rheumatologist, oncologist, neurosurgeon, physiotherapist, psychologist). The mean pain duration was 168.99 months $(S D=111.74)$ and almost every patient indicated having more than one pain location $(M=$ $3.81, S D=1.89$; range 1-9). Most frequently reported pain locations were the back $(92.9 \%)$, neck $(67.1 \%)$ and leg $(66.7 \%)$. Mean pain severity was 3.86 as measured by the MPI $(S D=0.97)$ and mean disability was 39.17 on the PDI ( $S D=11.34$ ); these mean levels compare well with previously reported data from chronic pain patients $\left(M_{\mathrm{MPI}}=4.2, S D_{M P I}=1.1 ; M_{\mathrm{PDI}}=44.6, S D_{P D I}=13.4\right)[10,38]$. On average, the level of daily PA was $2.46(S D=1.24)$ and the level of daily NA was $1.75(S D=1.12)$. The mean level of instability for PA was $1.08(S D=0.98)$ and for NA $1.06(S D=1.01)$, indicating that, on average, patients' PA and NA levels varied by approximately 1 point (on a scale from 0 to 6) from one day to the next. 
Those with higher (+1 SD) levels of instability showed differences of approximately 2 scale points in their affect levels on successive days, whereas patients' with lower (-1 SD) levels of instability had near to zero differences in their affect levels from day-to-day. Mean scores for state $(M=38, S D=9.49)$ and trait anxiety $(M=47.17, S D=11.35)$ were comparable with those in other chronic pain studies $[3,13]$. Depression scores $(M=8.39, S D=4.03)$ were mildly elevated compared to available norms [48].

\subsection{Correlation analyses}

Pearson correlations were calculated between NA and PA instability, assessed via end-of-day diary, and measures assessed via questionnaires (i.e. depression, state anxiety, trait anxiety, baseline pain severity, baseline disability). Further Pearson correlations were calculated between NA and PA instability and the mean levels of daily PA and NA, measures based on the daily reported emotions. The two measures of affective instability were not related to the mean levels of daily affect. Further, NA instability was related to more trait anxiety (STAI), more pain (MPI) and more disability (PDI). For PA instability there were no significant relationships, except with NA instability. An overview of the means and correlations is presented in Table 1.

- $\quad$ Table 1 about here -

\subsection{Multilevel analyses}

Separate multilevel models were run with daily disability and daily cognitive complaints as outcome measures. We predicted that NA instability would be positively related to daily disability and daily cognitive complaints and moderate the relationship between daily pain severity and its outcomes. We derive similar hypotheses to investigate the relationship with PA instability.

\subsubsection{NA instability}

\section{Daily disability}

Initial analyses indicated that there was substantial variance in reported daily disability between $(43 \%)$ and within (57\%) patients. First, we included daily pain severity as a Level 1 predictor. The model proved to be better $\left(X^{2}(3)=367.50, P<.001\right)$ than the model without any predictor. Second, we added NA instability as a Level 2 predictor, and additional Level 2 measures, i.e. age, gender, pain duration, baseline pain severity, pain disability and the mean daily level of NA, as control variables. The proposed model proved to be better than the model with only daily pain severity as a Level 1 predictor $\left(X^{2}(7)=\right.$ 
44.18, $P<.001)$. Only the mean level of daily NA was a significant predictor for the intercept of daily disability (coefficient $=.895, t(62)=5.5, P<.001$ ), indicating that patients reporting more daily NA also reported more daily disability. We, therefore, excluded age, gender, pain duration, baseline pain severity and pain disability. Third, we included the interaction between NA instability (Level 2) and daily pain severity (Level 1) as a cross-level moderator of the Level 1 intercept and slope. The resulting model was better than without the cross-level interaction term $\left(X^{2}(3)=.847, P=.037\right)$. NA instability was a significant predictor for the Level 1 intercept, meaning the mean level of daily disability (coefficient $=$ $.445, t(67)=2.27, P=.026$ ), and the Level 1 slope (coefficient $=.094, t(67)=2.09, P=.041$ ), indicating a stronger within-person association between daily pain severity and disability for patients who were more unstable in their NA. The final model is shown in Table 2. It shows that (1) the mean level of daily NA and NA instability are related to daily disability and (2) NA instability moderates the association between daily pain severity and daily disability. Some further analyses were performed to test the robustness of our findings. For example, when including trait anxiety as a Level 2 variable, there were no significant changes for the predictive values of the Level 2 variables.

\section{Daily cognitive complaints}

Initial analyses indicated the presence of substantial variance in reported daily cognitive complaints between $(60 \%)$ and within $(40 \%)$ patients. First, we investigated the predicting effect of daily pain severity at Level 1 . The resulting model proved to be better $\left(X^{2}(3)=70.13, P<.001\right)$ than the model without any predictor. Second, we added NA instability as a Level 2 predictor, as were control variables, i.e. age, gender, pain duration, baseline pain severity and the mean level of daily NA. This model was better than the model with daily pain severity as single Level 1 predictor $\left(X^{2}(6)=28.06, P<.001\right)$. Only the mean level of daily NA was a significant predictor for the intercept (coefficient $=.77, t(63)=3.94, P$ $<.001$ ), indicating that patients reporting more daily NA also reported a higher level of daily cognitive complaints. Age, gender, pain duration and baseline pain severity were excluded from the model as they did not show any significant effects. Third, we added the interaction between NA instability (Level 2) and daily pain severity (Level 1) as a cross-level moderator of the Level 1 intercept and slope to the model. The model proved to be better than without cross-level interaction $\left(X^{2}(2)=8.73, P=.013\right)$. NA instability was a significant predictor only for the Level 1 slope (coefficient $=.13, \mathrm{t}(67)=2.39, P=.020$ ), indicating a stronger within-person association between daily pain severity and cognitive complaints for patients who were more unstable in their NA. The final model (Table 2) suggests that (1) the mean level of daily NA is related to daily cognitive complaints and (2) NA instability moderates the association between daily pain severity and daily cognitive complaints. The robustness of these findings was tested by some further analyses. For example, when including trait anxiety as a Level 2 variable, trait anxiety showed to be a 
significant predictor (coefficient $=.63, t(66)=2.12, P=.038$ ), but not the mean level of daily NA (coefficient $=.39, t(66)=1.48, P=.145)$. There was no change in the moderating role of NA instability.

In a series of posthoc-analyses we explored whether the effect of affective instability was moderated by age. Age only moderated the effect of NA in the model predicting cognitive complaints. Specifically, there was a significant interaction between age and NA instability on the within-person association between daily pain and cognitive complaints (coefficient $=-.185, t(65)=-2.34, P=.023$ ), meaning that the effect of NA instability was larger in younger patients. Age did not moderate the effects of affective instability in any of the other three models $(P \mathrm{~s}>.33)$.

- Table 2 about here -

\subsubsection{PA Instability}

\section{Daily disability}

Initial analyses indicated that there was substantial variance in reported daily disability between and within patients, and daily pain severity to be a significant Level 1 predictor (see above). In a next step, we added PA instability as a Level 2 predictor and further Level 2 control variables, i.e. age, gender, pain duration, baseline pain severity, baseline pain disability and the mean level of daily PA, to our model. The proposed model proved to be better than the model with only daily pain severity as Level 1 predictor $\left(X^{2}\right.$ $(7)=32.02, P<.001)$. Only the mean level of daily PA was a significant predictor for the Level 1 intercept (coefficient $=-.69, t(62)=-4.15, P<.001)$, reflecting the fact that patients reporting more daily PA showed less daily disability. Age, gender and pain duration, baseline pain severity and disability were dropped from the model, because they were not significant. In a last step, we included the interaction between PA instability (Level 2) and daily pain severity (Level 1) as a cross-level moderator of the Level 1 intercept and slope. Although pointing in the same direction, the model failed to reach significance $\left(X^{2}\right.$ $(2)=3.43, P=.178$ ). The final model (Table 3 ) shows that the mean level of daily PA is related to daily disability. Including trait anxiety at Level 2 , showed trait anxiety to be a significant predictor (coefficient $=.46, t(66)=2.28, P=.026)$, similar to the mean level of daily PA (coefficient $=-.63, t(66)=-2.88, P=$ $.005)$.

\section{Daily cognitive complaints}

Initial analyses indicated that there was substantial variance in reported daily cognitive complaints between and within patients, and daily pain severity to be a significant Level 1 predictor (see above). In a next step, we included PA instability as a Level 2 predictor, as were control variables i.e. age, gender, pain duration, pain severity at baseline and the mean level of daily PA. The proposed model proved to be better 
than the model with only daily pain severity as Level 1 predictor $\left(X^{2}(6)=18.38, P=.006\right)$. The mean level of daily PA (coefficient $=-.48, t(63)=-2.43, P=.018)$ and baseline pain severity (coefficient $=$ $.435, t(63)=2.04, P=.046)$ were significant predictors for the intercept, indicating that patients reporting higher levels of daily PA or lower levels of baseline pain severity reported less cognitive complaints in daily life. Due to the non-significant effects of age, gender and pain duration, they were dropped from the model. In a last step, we entered the interaction between PA instability (Level 2) and daily pain severity (Level 1) as a cross-level moderator of the Level 1 intercept and slope. The resulting model was not better than without the cross-level interaction term $\left(X^{2}(2)=.860, P>.500\right)$. Results of the final model are summarized in Table 3. They reveal that the mean level of daily PA is related to daily cognitive complaints. When including trait anxiety as a Level 2 variable, it proved to be a significant predictor (coefficient $=.78, t(65)=3.15, P=.002$ ), whereas the significant predictive effect of the mean level of daily PA of $P=.028$ changed to $P=.857$.

Additional analyses including depression scores (HADS) showed that despite a positive relation to mean levels of daily pain severity, disability and cognitive complaints (correlations ranging from .35 to .43), depression did not reliably moderate the within-person associations between daily pain and either disability or cognitive complaints $(P s>.18)$. NA instability remained a significant moderator of the associations between daily pain and both disability and cognitive complaints $(P s<.03)$ even after controlling for depressive symptoms. PA instability became a statistically significant moderator of the association between daily pain and disability $(P=.024)$.

- Table 3 about here -

\section{Discussion}

This study investigated the role of affective instability in the daily experience of pain, disability and cognitive complaints using an end-of-day diary over 14 days. The results can be readily summarized. First, the results show a significant relationship between mean levels of daily affect and pain severity and disability, confirming previously reported results about the relationship between NA, PA and pain $[1,41,62]$. Second, NA, but not PA, instability predicted daily disability over and above the mean level of daily NA, and even after controlling for age, gender, baseline pain severity, pain chronicity and trait anxiety. Third, NA instability moderated the relationship between daily pain severity and pain-related 
outcomes: Daily pain severity was more strongly associated with daily disability and cognitive complaints in patients with higher levels of NA instability.

This study is the first to reveal the role of NA instability in the adjustment to chronic pain. Our findings demonstrate that not only the intensity but also the time course and variability of NA is related to daily pain outcomes. As this study is the first of its kind, research will need to further corroborate our findings, and also investigate underlying processes. One explanation for current findings may be found in the type and quality of emotion regulation skills. Indeed, affective instability has previously been interpreted as reflecting a dysfunctional regulation of emotions [8,47]. Although this view mostly stems from the literature on psychopathology, similar processes may play a role when for chronic pain patients have to cope coping with the daily demands of pain [17]. It may well be that individuals who have problems with regulating NA also have difficulties to cope with pain and, thus, experience more interference by pain in daily life. This interference by pain may result in disability and cognitive complaints. If this is true, there may be a strong overlap in regulating pain and regulating NA. This may be a reasonable assumption as pain involves a sensory and an emotional experience. Also, Linton argued for commonalities in problems of regulation in chronic pain and emotional disorders [30]. An important strategy for clinicians may then be to target NA instability. As yet we do not know how this is best accomplished. If there are deficits in particular regulation skills, interventions may include skills training, such as the Affect Regulation Training [6], which is a module-based intervention to improve emotion regulation skills. If, on the other hand, there is evidence for rigid overregulation, mindfulness training $[21,43]$ or Acceptance and Commitment Therapy [23,35,61] may be promising approaches.

A remarkable finding of our study is that NA instability but not PA instability predicted painrelated outcomes, although the association between NA and PA instability was high. This pattern of results is not unusual. Previous research has shown a similar relationship between instability measures of PA and NA [28,32]. These findings may be explained by shared method variance between instability measures of NA and PA: They are based on similar mathematical algorithms. The finding, however, that NA instability, but not PA instability, predicts pain-related outcomes is in line with previous research in psychopathology investigating affective instability in natural contexts $[25,55,56]$. For example, depressed patients show higher NA instability, but not PA instability, compared to healthy volunteers [55].

At first sight, our findings are not in line with the predictions of the dynamic model of affect [14]. This model states that NA and PA are relatively independent during non-stressful periods, whereas they merge to one bipolar dimension under stressful or painful conditions. From this perspective, one might have expected a similar pattern of results for NA and PA instability. Nevertheless, although a relatively strong relationship was found between the mean levels of NA and PA instability, only NA instability was found to moderate the relationship between pain severity and disability or cognitive complaints. One 
explanation for not finding such a pattern for PA may relate to the fact that there are fewer fluctuations in PA. This is, however, not the case in our study: The level of instability was similar for NA and PA. Another explanation may be that our measure of affective instability captures a unique parameter that is not addressed by the dynamic model of affect. In line with this argument are the findings that NA instability and PA instability are not related to the mean levels of daily NA and PA.

Our findings corroborate the notion that affective instability does not measure the intensity of emotional experiences, but rather their temporal variability. Recently, the examination of the dynamics of affect has increased in order to improve our understanding of psychological maladjustment and psychopathology $[15,25,28,55,56]$. Within this emerging field, emotional responding reflects a dynamic process that takes place in response to changing contextual demands. Thus, emotional responding that is adaptive, is believed to be flexible, rather than rigid or stereotypical [54]. In case of affective instability, an individual tends to show frequent mood shifts with a transient, fluctuating course [56]. The distinction between intensity and temporal variability completes the picture of dynamically fluctuating emotions in daily life and demonstrates the importance of broadening the perspective in order to better understand daily emotional experiences. In addition, we found that NA instability, but not PA instability, is related to baseline pain severity and baseline disability. This finding expands upon previous research relating affective instability to mental disorders $[16,55,56]$ and furthers our understanding of daily functioning, i.e. disability and cognitive complaints, of chronic pain patients.

In consideration of affective instability as a reflection of dysfunctional emotion regulation $[8,47]$, we also investigated the relationship between affective instability and measures of emotional functioning, i.e. depressive mood and trait anxiety. We only found a negative association between NA instability and trait anxiety, which is in line with the model of Hofmann and colleagues (2012), which links dysregulation of NA to anxiety disorders [24]. Nevertheless, it is important to note that including trait anxiety in our models did not affect the effects of NA instability. Although not the focus of the current study, one might further have expected a relationship between depressive mood and affective instability. Hamilton and colleagues (2005) suggest that affect regulation may influence psychological functioning in patients with chronic pain [22]. They further assume that the reason for the relationship between affect regulation and adjustment to pain may be due to the fact that these patients are more vulnerable to depression. In the current study, however, we neither found a relationship between affect dysregulation (i.e. affective instability) and depression, nor did the inclusion of depressive symptoms in our models change the predictive and moderating role of NA instability for daily disability and cognitive complaints. More research is needed in this regard. Nevertheless, in line with numerous studies reporting high levels of emotional distress in chronic pain patients $[4,33,34]$, we found a strong association between baseline depressive mood, anxiety and pain severity. 
This study has some limitations. First, we did not include a control group and therefore we cannot compare our findings with a non-clinical sample. Second, we investigated a heterogeneous group of chronic pain conditions so that findings cannot be ascribed to specific conditions. Third, the analyses are based on end-of-day diaries, assessing fluctuations in affect over days. Our results may be further corroborated by also studying within-day emotional fluctuations. Fourth, neither did we have data on putative mediating variables such as emotion regulation strategies used, or indicators of failed regulation such as perseverative cognitions (i.e. worry and rumination) [18], nor did we include other variables known to influence levels of pain-related disability (e.g. pain-related fear) in the analysis. Fifth, the effect of affective instability may be moderated by other variables. We found such an effect for age, albeit in posthoc-analyses. This finding suggests that emotional experiences may grow more stable with age [9]. Sixth, our measure of affective instability captures the temporal fluctuations of emotions over 14 days. Nevertheless, it remains unclear which individual or contextual factors may underlie, or be related to, this process. Seventh, there is a strong tradition to psychometrically validate questionnaires. The validation of diary items is still in its infancy. Although items in the current study were carefully selected and piloted for understanding and feasibility in patients with chronic pain, validation of the diary items should be further developed (e.g. by using cognitive interviewing [11]). Eighth, our analyses are best considered as cross-sectional. We did not perform time lag analyses. Therefore, one should be careful in inferring causality between variables. Finally, we only focus upon affective instability as a marker of dysfunctional regulation. Other measures are possible, such as psychophysiological measures of emotion regulation capacity (i.e. heart rate variability), and their use may further our understanding of their role in the context of chronic pain.

\section{Conflict of interest statement}

There are no conflicts of interest that may arise as a result of this research.

\section{Acknowledgements}

The authors want to thank Emelien Lauwerier and Lies Notebaert for their help in the data collection. Preparation of this paper was partly supported by the University of Luxembourg, Grant BOF/GOA2006/001 of Ghent University and FWO project G.017807. 


\section{References}

[1] Affleck G, Tennen, H, Urrwos S, Higgins P. Person and contextual features of daily stress reactivity: individual differences in relations of undesirable daily events with mood disturbance and chronic pain intensity. J Pers Soc Psychol 1994;66:329-40.

[2] Attridge N, Crombez G, Van Ryckeghem D, Keogh E, Eccleston C. The experience of cognitive intrusion of pain: scale development and validation. PAIN 2015;156:1978-90.

[3] Asmundson GJG, Carleton NR, Ekong J. Dot-probe evaluation of selective attentional processing of pain cues in patients with chronic headaches. PAIN 2005;114:250-6.

[4] Bair MJ, Robinson RL, Katon W, Kroenke K. Depression and pain comorbidity: a literature review. Arch Intern Med 2003;162:2433-45.

[5] Barnes LLB, Harp D, Jung WS. Reliability generalization of scores on the Spielberger State-Trait Anxiety Inventory. Educ Psychol Meas 2002;62:603-18.

[6] Berking M, Whitley B. Affect regulation training: a practitioner's manual. New York: Springer, 2014.

[7] Bolger N, Laurenceau JP. Intensive longitudinal methods: an introduction to diary and experience sampling research. New York: Guilford, 2013.

[8] Carpenter RW, Trull TJ. Components of emotion dysregulation in borderline personality disorder: a review. Curr Psychiatry Rep 2013;15:335.

[9] Carstensen LL, Turan B, Scheibe S, Ram N, Ersner-Hershfield H, Samanez-Larkin GR, Brooks KP, Nesselroade JR. Emotional experience improves with age: evidence based on over 10 years of experience sampling. Psychol Aging 2011;26:21-33.

[10] Chibnall JT, Tait RC. The pain disability index: factor structure and normative data. Arch Phys Med Rehabil 1994;75:1082-6.

[11] Collins D. Pretesting survey instruments: an overview of cognitive methods. Qual Life Res 2003;12:229-38.

[12] Connelly M, Keefe FJ, Affleck G, Lumley MA, Anderson T, Waters S. Effects of day.to-day affect regulation on the pain experience of patients with rheumatoid arthritis. PAIN 2007;131:162-70. 
[13] Crombez G, Hermans D, Adriaensen H. The emotional Stroop task and chronic pain: what is threatening for chronic pain sufferers? Eur J Pain 2000;4:37-44.

[14] Davis MC, Zautra AJ, Smith BW. Chronic pain, stress, and the dynamics of affective differentiation. J Pers 2004;72:1133-59.

[15] Ebner-Priemer UW, Eid M, Kleindienst N, Stabenow S, Trull TJ. Analytic strategies for understanding affective (in)stability and other dynamic processes in psychopathology. J Abnorm Psychol 2009;118:195-202.

[16] Ebner-Priemer UW, Trull TJ. Ecological momentary assessment of mood disorders and mood dysregulation. Psychol Assess 2009;21:463-75.

[17] Eccleston C, Crombez G. Pain demands attention: a cognitive-affective model of the interruptive function of pain. Psychol Bull 1999;125:356-66.

[18] Eccleston C, Crombez G. Worry and chronic pain: a misdirected problem solving model. PAIN 2007;132:233-6.

[19] Enders, CK, Tofighi D. Centering predictor variables in cross-sectional multilevel models: a new look at an old issue. Psychol Methods 2007;12:121.

[20] Gross JJ, Jazaieri H. Emotion, emotion regulation, and psychopathology: an affective science perspective. Clin Psychol Sci 2014;2:387-401.

[21] Grossman P, Tiefenthaler-Gilmer U, Raysz A, Kesper U. Mindfulness training as an intervention for fibromyalgia: evidence of postintervention and 3-year follow-up benefits in well-being. Psychother Psychosom 2007;76:226-33.

[22] Hamilton NA, Zautra AJ, Reich JW. Affect and pain in rheumatoid arthritis: do individual differences in affective regulation and affective intensity predict emotional recovery from pain? Ann Behav Med 2005;29:216-24.

[23] Hayes SC, Strosahl, K, Wilson KG. Acceptance and Commitment Therapy. New York: Guilford Press, 1999. 
[24] Hofmann SG, Sawyer AT, Fang A, Asnaani A. Emotion dysregulation model of mood and anxiety disorders. Depress Anxiety 2012;29:409-16.

[25] Houben M, Van Den Noortgate W, Kuppens P. The relation between short-term emotion dynamics and psychological well-being: a meta-analysis. Psychol Bull 2015;141:901-30.

[26] Jahng S, Wood PK, Trull TJ. Analysis of affective instability in ecological momentary assessment: indices using successive difference and group comparison via multilevel modeling. Psychol Methods 2008;13:354-7.

[27] Kerns RD, Turk DC, Rudy TE. The West Haven-Yale Multidimensional Pain Inventory. PAIN $1985 ; 23: 345-56$.

[28] Koval P, Ogrinz B, Kuppens P, Van den Bergh O, Tuerlinckx F, Sütterlin S. Affective instability in daily life is predicted by resting heart rate variability. PLoS ONE 2013;8:1-10.

[29] Kreft I, de Leeuw J. Introducing multilevel modelling. London: Sage Publications, 1998.

[30] Linton SJ. A transdiagnostic approach to pain and emotion. J Appl Biobehav Res 2013;18:82-103.

[31] Lousberg R, Van Breukelen GJP, Groenman NH, Schmidt AJM, Arntz A, Winter FAM. Psychometric properties of the Multidimensional Pain Inventory, Dutch language version (MPIDLV). Behav Res Ther 1999;37:167-82.

[32] Maciejewski DF, van Lier PA, Branje SJ, Meeus WH, Koot HM. A 5-year longitudinal study on mood variability across adolescence using daily diaries. Child Dev 2015;86:1908-21.

[33] McCracken LM, Faber SD, Janeck AS. Pain-related anxiety predicts non-specific physical complaints in persons with chronic pain. Behav Res Ther 1998;36:621-30.

[34] McCracken LM, Iverson GL. Predicting complaints of impaired cognitive functioning in patients with chronic pain. J Pain Symptom Manage 2001;21:392-6.

[35] McCracken LM, Vowles KE, Eccleston C. Acceptance-based treatment for persons with complex, long standing of chronic pain: a preliminary analysis of treatment outcome in comparison to a waiting phase. Behav Res Ther 2004;43:1335-46. 
[36] Morley S, Eccleston C, Williams A. Systematic review and meta-analysis of randomized controlled trials of cognitive behaviour therapy and behaviour therapy for chronic pain in adults, excluding headache. PAIN 1999;80:1-13.

[37] Nezlek JB. Multilevel modeling analyses of diary-style data. In: Mehl MR, Conner TS, editors. Handbook of research methods for studying daily life. New York: Guilford Press; 2012. pp.357383.

[38] Nicholas MK, Asghari A, Blyth FM. What do the numbers mean? Normative data in chronic pain measures. PAIN 2008;134:158-173.

[39] Parenteau SC., Haythornthwaite JA. Assessment of functioning and disability in pain syndromes. In: Ebert MH, Kerns RD. Behavioral and psychopharmacologic pain management. New York: Cambridge University Press; 2011. pp. 67-81.

[40] Pollard CA. Preliminary validity study of the Pain Disability Index. Percept Mot Skills 1984;59:974.

[41] Potter PT, Zautra AJ, Reich JW. Stressful events and information processing dispositions moderate the relationship between positive and negative affect: implications for pain patients. Ann Behav Med 2000;22:191-8.

[42] Raudenbush SW, Bryk AS. Hierarchical linear models. Applications and data analysis methods. Newbury Park: CA Sage; 2002.

[43] Rosenzweig S, Greeson JM, Reibel DK, Green JS, Jasser SA, Beasley D. Mindfulness-based stress reduction for chronic pain conditions: variation in treatment outcomes and role of home meditation practice. J Psychosom Res 2010;68:29-36.

[44] Roth RS, Geisser ME, Theisen-Goodvich M, Dixon P. Cognitive complaints are associated with depression, fatigue, female sex, and pain catastrophizing in patients with chronic pain. Arch Phys Med Rehabil 2005;86:1147-54.

[45] Rudy TE. Multiaxial assessment of pain. Multidimensional Pain Inventory. User's manual. Pittsburgh, PA: Departments of Anaesthesiology and Psychiatry and Pain Evaluation and Treatment Institute, University of Pittsburgh School of Medicine, 1989. 
[46] Russell JJ, Moskowitz DS, Zuroff DC, Sookman D, Paris J. Stability and variability of affective experience and interpersonal behavior in borderline personality disorder. J Abnorm Psychol 2007;116:578-88.

[47] Selby EA, Cornelius T, Fehling KB, Kranzler A, Panza EA, Lavender JM, Wonderlich SA, Crosby RD, Engel SG, Mitchell JE, Crow SJ, Peterson CB, Le Grange D. A perfect storm: examining the synergistic effects of negative and positive emotional instability on promoting weight loss activities in anorexia nervosa. Front Psychol 2015;6:1260.

[48] Snaith RP, Zigmond AS. HADS: Hospital Anxiety and Depression Scale. Windsor, UK: NFER Nelson, 1994.

[49] Solberg Nes L, Roach AR, Segerstrom SC. Executive functions, self-regulation, and chronic pain: a review. Ann Behav Med 2009;37:173-83.

[50] Spielberger CD. Manual for the State-Trait Anxiety Inventory. Palo Alto, CA: Consulting Psychologists Press, 1983.

[51] Spielberger CD, Gorsuch RL, Lushene RE. Manual for the State-Trait Anxiety Inventory. Palo Alto, CA: Consulting Psychologists Press, 1970.

[52] Strand EB, Zautra AJ, Thoresen M, Odegard S, Uhlig T, Finset A. Positive affect as a factor of resilience in the pain-negative affect relationship in patients with rheumatoid arthirits. J Psychosom Res 2006;60:477-84.

[53] Sturgeon JA, Zautra AJ, Arewasikporn A. A multilevel structural equation modeling analysis of vulnerabilities and resilience resources influencing affective adaptation to chronic pain. PAIN $2014 ; 155: 292-8$.

[54] Thompson RA. Emotion regulation: a theme in search of definition. Monogr Soc Res Child Dev 1994;59:25-52.

[55] Thompson RJ, Mata J, Jaeggi SM, Buschukuehl M, Jonides J, Gotlieb IH. The everyday emotional experience of adults with major depressive disorder: examining emotional instability, intertia, and reactivity. J Abnorm Psychol 2012;121:819-29. 
[56] Trull TJ, Solhan MB, Tragesser SL, Jahng S, Wood PK, Piasecki TM, Watson D. Affective instability: Measuring a core feature of borderline personality disorder with ecological momentary assessment. J Abnorm Psychol 2008;117:647-61.

[57] Van Damme S, Crombez G, Eccleston C. Keeping pain in mind: a motivational account of attention to pain. Neurosci Biobehav R 2010;34:204-13.

[58] Van der Ploeg HM, Defares PB, Spielberger CD. Handleiding bij de Zelf-Beoordelings Vragenlijst, ZBV. Een Nederlandstalige bewerking van de Spielberger State-Trait Anxiety Inventory, STATDY. Lisse, The Netherlands: Swets en Zeitlinger, 1980.

[59] Van Ryckghem DML, Crombez G, Goubert L, De Houwer J, Onraedt T, Van Damme S. The predictive value of attentional bias towards pain-related information in chronic pain patients: a diary study. PAIN 2013;154:468-75.

[60] Veirman E, Fontaine JRJ. Revisiting the dimensional structure of the emotion domain. Cogn Emot 2014:1-16.

[61] Vowles KE, McCracken LM, O’Brien JZ. Acceptance and values-based action in chronic pain: a three-year follow-up analysis of treatment effectiveness and process. Behav Res Ther 2011;49:74855.

[62] Zautra AJ, Burleson MH, Smith CA, Blalock SJ, Wallston KA, DeVellis RF, DeVellis BM, Smith TW. Arthritis and perceptions of quality of life: an examination of positive and negative affect in rheumatoid arthritis patients. Healthy Psychol 1995;14:399-408.

[63] Zautra AJ, Johnson JL, Davis MC. Positive affect as a source of resilience for woman with chronic pain. J Consult Clin Psychol 2005;73:212-20.

[64] Zautra AJ, Smith B, Affleck B, Tennen H. Examinations of chronic pain and affect relationships: applications of a dynamic model of affect. J Consult Clin Psych 2001;69:786-95.

[65] Zigmond AS, Snaith RP. The Hospital Anxiety and Depression Scale. Acta Psychiatr Scand $1983 ; 67: 361-70$. 
Table 1

Means $(M)$, standard deviations $(S D)$ and Pearson correlation coefficients for all measures.

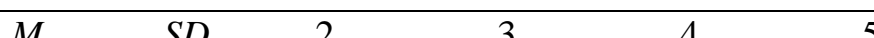

\begin{tabular}{|c|c|c|c|c|c|c|c|c|c|c|}
\hline 1. Instability of PA (MSSD) & 1.08 & 0.98 & $.683^{* * *}$ & -.053 & .008 & .069 & .194 & .151 & .028 & -.043 \\
\hline 2. Instability of NA (MSSD) & 1.06 & 1.01 & & .069 & .058 & $.284^{*}$ & $.313^{* *}$ & $.304^{*}$ & -.120 & .088 \\
\hline 3. Depression (HADS-D) & 8.4 & 4.03 & & & $.529^{* * * *}$ & $.749^{* * *}$ & $.463^{* * *}$ & $.331^{* *}$ & $-.679^{* * *}$ & $.690^{* * * *}$ \\
\hline 4. State anxiety (STAI-S) & 38 & 9.49 & & & & $.609^{* * *}$ & .184 & -.024 & $-.408^{* *}$ & $.618^{* * *}$ \\
\hline 5. Trait anxiety (STAI-T) & 47.17 & 11.35 & & & & & $.402^{* *}$ & $.286^{*}$ & $-.599^{* * *}$ & $.777^{* * *}$ \\
\hline 6. Pain severity (MPI) & 3.86 & 0.97 & & & & & & $.554^{* * * *}$ & $-.350^{* *}$ & $.376^{* *}$ \\
\hline 7. Disability (PDI) & 39.17 & 11.34 & & & & & & & $-.252^{*}$ & $.260^{*}$ \\
\hline 8. Mean daily PA & 2.46 & 1.24 & & & & & & & & $-.585^{* * *}$ \\
\hline 9. Mean daily NA & 1.75 & 1.12 & & & & & & & & \\
\hline
\end{tabular}

PA, positive affect; NA, negative affect; MSSD, Mean Square Successive Difference; HADS-D, Depression scale of the Hospital Anxiety and Depression Scale; STAI-S, State subscale of the State-Trait Anxiety Inventory; STAI-T, Trait subscale of the State-Trait Anxiety Inventory; MPI, Multidimensional Pain Inventory; PDI, Pain Disability Index, HADS-D, $\mathrm{n}=67$.

$\begin{array}{rl}{ }^{*} P & <.05 \\ { }^{* *} & P<.01 \\ { }^{* * *} P & <.001\end{array}$ 
Table 2

Final hierarchical linear models in regard of NA instability.

\begin{tabular}{llll}
\hline Dependent variable & Coefficient & SE & $T$ \\
(diary items) & & &
\end{tabular}

Daily disability

$\begin{array}{lccc}\text { Intercept }(\beta 00) & 4.484 & 0.16 & 27.549^{* * *} \\ \text { NA_MSSD }(\beta 01) & 0.445 & 0.20 & 2.272^{*} \\ \text { Mean NA ( } \beta 02) & 1.020 & 0.17 & 5.856^{* * *} \\ \text { Daily pain severity ( } \beta 10) & 0.916 & 0.05 & 18.457^{* * *} \\ \text { Daily pain severity x NA_MSSD ( } \beta 11) & 0.094 & 0.04 & 2.085^{*} \\ & & & \\ \text { Daily pain severity x Mean NA ( } \beta 12) & 0.022 & 0.05 & 0.411\end{array}$

Daily cognitive

complaints

$\begin{array}{lccc}\text { Intercept }(\beta 00) & 3.233 & 0.17 & 18.635^{* * * *} \\ \text { NA_MSSD }(\beta 01) & 0.238 & 0.16 & 1.521 \\ \text { Mean NA ( } \beta 02) & 0.872 & 0.16 & 5.427^{* * *} \\ \text { Daily pain severity }(\beta 10) & 0.277 & 0.05 & 5.395^{* * * *} \\ \text { Daily pain severity x NA_MSSD }(\beta 11) & 0.128 & 0.05 & 2.386^{*} \\ & & & \\ \text { Daily pain severity x Mean NA }(\beta 12) & 0.070 & 0.04 & 1.630\end{array}$

NA, negative affect; MSSD, Mean Square Successive Difference.

\footnotetext{
${ }^{*} P<.05$

${ }^{* *} P<.01$

${ }^{* * *} P<.001$
} 
Table 3

Final hierarchical linear models in regard of PA instability.

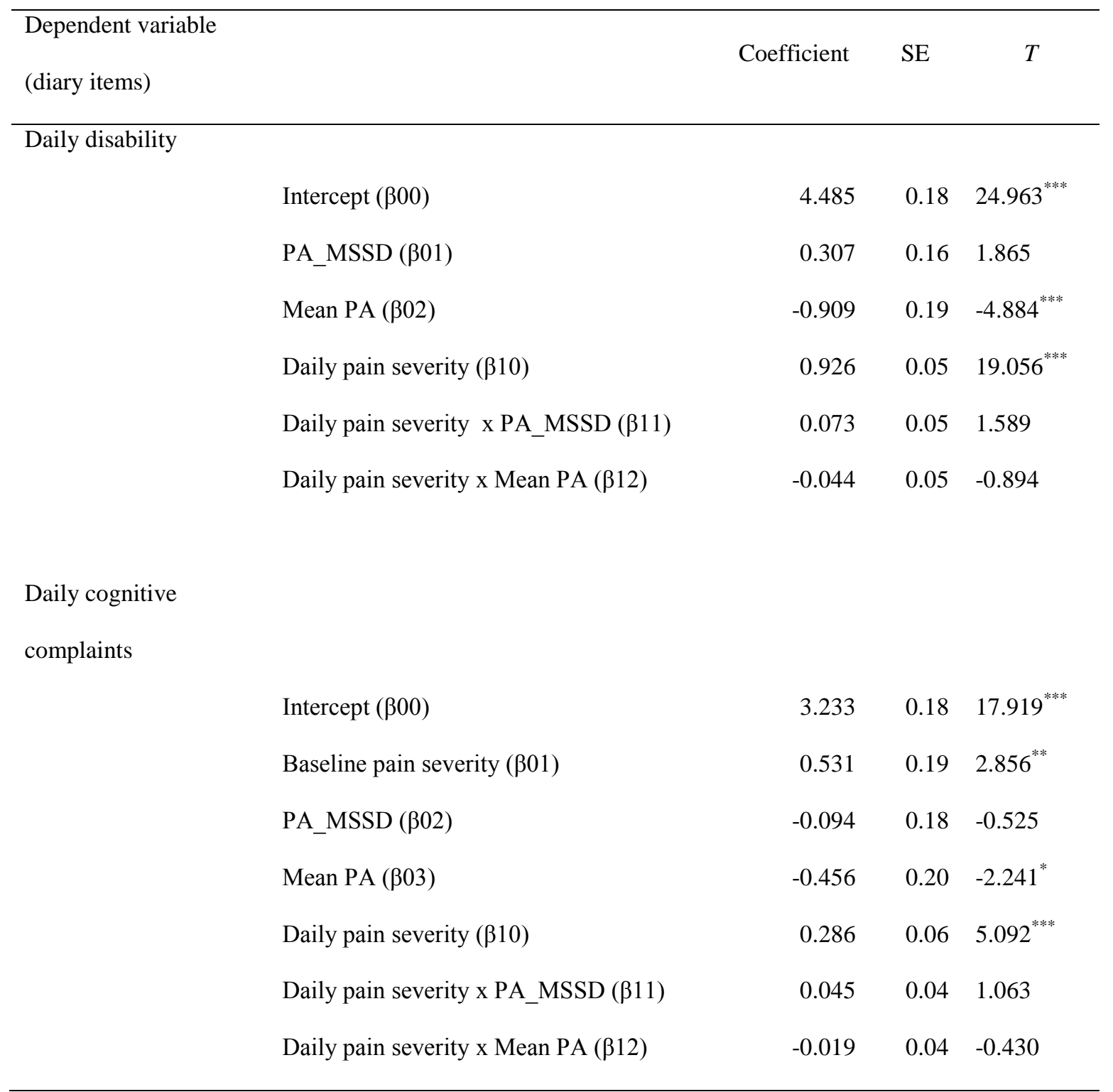

Note. PA, positive affect; MSSD, Mean Square Successive Difference.

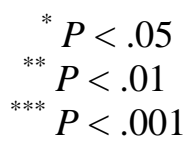

